\title{
Is clinical neurology really so difficult?
}

\section{F Schon, P Hart, C Fernandez}

\section{Neurosciences need to be made more accessible for medical students}

$\mathrm{N}$ eurology, it seems, has a reputation among medical specialties of being particularly difficult. This was highlighted in the British Medical Journal in $1999^{1}$ when the editor wrote, "the neurologist is one of the great archetypes: a brilliant, forgetful man with a bulging cranium....who....talks with ease about bits of the brain you'd forgotten existed, adores diagnosis and rare syndromes, and-most importantly-never bothers about treatment." The relevance of this view to the teaching of neurology was analysed in a not totally serious letter in 1994 by Ralph Jozefowicz ${ }^{2}$ entitled "Neurophobia," in which the author claimed $50 \%$ of medical students at some stage have " a fear of neural sciences and clinical neurology." His explanation for this was students' inability to apply knowledge of basic sciences to the clinical situation. This letter was based on personal views not supported by what the $B M J$ called "evidence based education" in another editorial in $1999 .^{3}$ We therefore set out to ascertain perceptions of seven major medical specialties among British medical students, senior house officers (SHOs), and general practitioners, principally in order to find out the actual perceptions of neurology in comparison with the other disciplines.

\section{THE STUDY}

Part 1

Part 1 was a questionnaire based study, given to four separate groups and the results presented are the analysis of 345 replies: 101 medical students from St George's Hospital (five sequential groups of about 20 at the end of their fourth year clinical neurology attachment), 85 medical students from the Royal Free Hospital attending a revision course for their final examinations ( the total size of this group was 94), 100 SHOs attending teaching courses for parts 1 and 2 of the MRCP examination (of a total of 108), and 59 general practitioners (3lof whom were attending a postgraduate neurology session and 28 were attending a meeting of general practitioners who teach medical students at St George's Hospital. All were asked three questions about seven medical specialties: cardiology, endocrinology, gastroenterology, geriatrics, neurology, respiratory medicine, and rheumatology. Question 1: What is your current level of interest?

Six options were offered: $0=$ not known or other; $1=$ little or no interest; $2=$ some interest; $3=$ moderate interest; $4=$ quite interested; $5=$ very interested.

Question 2: What is your current level of knowledge?

The six options offered were: $0=$ not known or other; $1=$ little or none; $2=$ some; $3=$ moderate; $4=$ fair 5 = great .

Question 3: Do you think the subject is easy or difficult?

The six options were: $0=$ not known or other; $\quad \mathrm{l}=$ very easy; $2=$ quite easy; $3=$ moderate; $4=$ quite difficult; $5=$ very difficult.

The 159 qualified doctors (SHOs and general practitioners), but not the students, were also asked the following fourth question:

Question 4: When you see a patient in accident and emergency (for the SHOs)/your surgery (for the general practitioners) with a not straight forward complaint in each of the following areas, what do you feel? $1=$ very uneasy; $2=$ uneasy; $3=$ averagely competent; $4=$ confident $; 5=$ very confident .

The data presented are the pooled results for all 345 questionnaires unless stated otherwise. For each of questions 1 to 4 , the difference between the mean scores for neurology and the other six specialties was examined using the independent sample $t$ test.

\section{Part 2}

After the results of the initial study had been analysed a second questionnaire study was carried out on another group of SHOs attending MRCP courses, aimed at trying to analyse why neurology was felt to be a difficult subject. Five possible reasons were selected:

1. The need to know basic neuroscience

2 . The complex clinical examination

3. Neurology having a reputation as a difficult subject

4. Neurology covering such a large number of diagnoses

5. Neurology being badly taught.

For each of these reasons, five options were offered: $0=$ do not know; $1=$ unimportant factor; 2 = possible factor; $3=$ important factor; $4=$ very impor tant factor.

At the end of the questionnaire there were two "open" questions:
1. Write why you think neurology is felt to be a hard subject.

2. Write ways in which you think neurology and neuroscience teaching could help the situation.

We analysed the replies of 80 SHOs (of a total of 96).

\section{RESULTS}

The answers to questions 2, 3, and 4 all showed how difficult neurology is perceived to be in comparison with the other six disciplines surveyed. When asked about their level of knowledge, the participants said they knew least neurology $(\mathrm{p}<0.005 v$ all other subjects $)$, followed by endocrinology and rheumatology. The subjects they felt they knew most about were respiratory medicine and gastroenterology (fig lA).

Second, not only did the whole group rank neurology as the most difficult subject but they felt it was far more difficult than any of the other subjects (fig 1B). This view was equally clear among all the four separate groups surveyed The perceived level of difficulty of neurology over all the other subjects was again highly significant $(\mathrm{p}<0.001)$. All the other subjects were ranked fairly close together, although gastroenterology was considered the easiest followed by geriatric medicine and respiratory medicine.

The two groups of qualified doctors who were also asked to assess their confidence in practical clinical situations felt clearly less confident in dealing with neurological cases than with any of the other medical specialties (fig lC) $(p<0.001)$. Both groups of doctors felt that elderly care cases were the easiest to handle, with respiratory medicine and gastroenterology almost as easy.

As a group, the 345 participants were not uninterested in neurology, ranking it third after cardiology and respiratory medicine, with gastroenterology fourth and geriatric medicine seventh (fig lD).

A further 80 SHOs were asked questions about why they felt neurology was perceived to be such a difficult subject. Five possible reasons were given for the ranking, and the responses to these were categorised on a scale of 1 to 4 , where $l=$ unimportant and $4=$ very important. The results are based on $78 \mathrm{com}-$ pleted questionnaires. "The need to know basic neuroscience" was ranked the most important factor (mean score 3.05), followed fairly closely by "poor teaching of neurology" (mean score 2.92 ). The "reputation of neurology as being difficult" and the "complexity of neurological examination" were ranked as moderate factors (mean scores 2.56 and 2.50 ), and the "wide range of diagnoses" was felt to be the least important (mean score 2.00).

The group of 78 SHOs also answered the two open questions given above in 
the section describing part 2 of the study. The full list of replies is given in table 1 .

The first open question was on why neurology was difficult. Eighty one reasons were given by the 70 people who answered the question. The most common reason was "poor teaching" followed closely by "problems related to neuroanatomy" and "the difficulty of the clinical examination." Six other common reasons were: concerns about neuroscience in general; the subject's awesome reputation; the complexity of the subject; the multiplicity of diagnoses; the lack of integrated clinical and basic neuroscience teaching; and finally the wide ranging nature of the subject.

The second open question asked for ways in which the situation could be improved by better neuroscience and neurology teaching. There were 78 responses to this question from 59 completed forms. Four main answers were given. The commonest was the need for more teaching, with half specifying the need for more clinical teaching. Next came the need to integrate basic neuroscience teaching with clinical neurology. Third was the need for better teaching, with half the respondents specifically mentioning neuroscience teaching. Fourth was the need to make clear what is "simple, basic, straightforward, and important".

\section{COMMENT}

In this questionnaire based survey of British medical students from two London teaching hospitals, SHOs, and general practitioners we examined perceptions of seven medical disciplines, with the aim of focusing on attitudes to neurology. We could not find any similar published surveys.

The main findings relate to how difficult neurology was felt to be. There were three separate aspects to this: first, neurology was ranked as far more difficult than any other discipline in a theoretical context; second, it was also the subject the doctors had least confidence in handling "at the bedside"; and third, neurology was the discipline the group felt least knowledgeable about.

This was a predictable response from the medical students; however, we were interested to find that it also applied to junior doctors and general practitioners, some of whom had been practising for 20 years. We appear to carry the perceptions formed at medical school throughout our subsequent careers.

The fact that neurology is thought to be so difficult does not, of course, mean that students or doctors actually know less neurology, do worse in neurology questions in examinations, or handle neurology cases less adequately in their clinics than they do the other six disciplines. They could, for example,
Table 1 Responses to the open questions

\begin{tabular}{llll}
\hline "Why is neurology difficult?" & & "How can teaching be improved?" \\
\hline Poor teaching & 17 & More teaching & 19 \\
Trouble with neuroanatomy & 16 & More integrated teaching & 17 \\
Trouble with clinical examination & 16 & Simple/basic teaching & 13 \\
Trouble with neuroscience & 11 & Better teaching & 13 \\
Hard reputation & 8 & More models/aids & 5 \\
Complexity of the subject & 7 & More revision sessions & 2 \\
Too many rare diagnoses & 6 & Simplify examination & 2 \\
Lack of integrated teaching & 6 & More neurology for SHOs & 1 \\
Wide ranging subject & 5 & More neuroanatomy & 1 \\
Non-neurologists teaching & 3 & Simpler textbooks & 1 \\
Need to visualise in three dimensions & 3 & Left blank & 19 \\
Not enough teaching time & 3 & Unclear comments & 4 \\
Not enough neurology for SHOs & 2 & & \\
Patients incurable & 1 & & \\
Left blank & 8 & & \\
Unclear comments & 10 & & \\
\hline
\end{tabular}
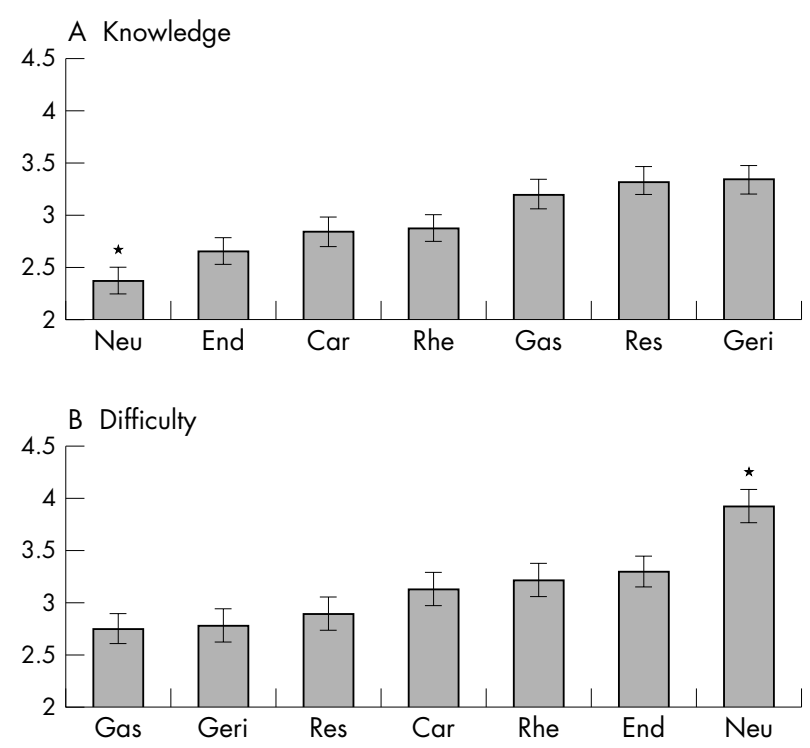

C Clinical confidence

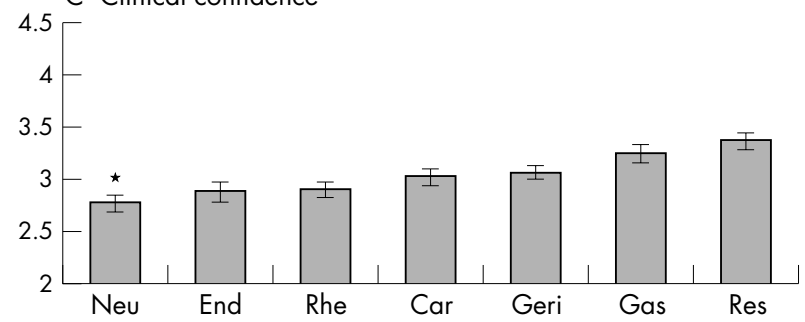

D Interest

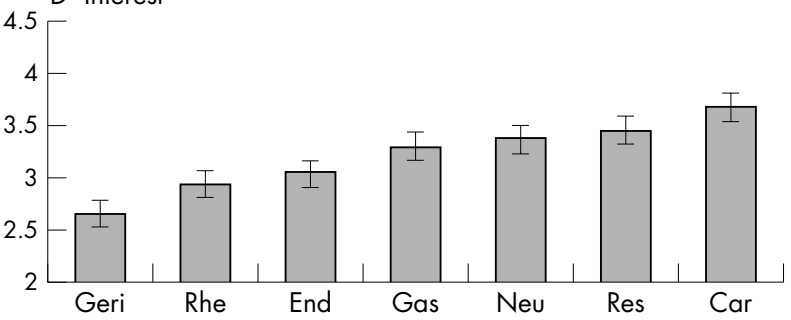

Figure 1 Histograms showing questionnaire results on seven medical disciplines. Panels A, $B$, and D are based on 345 replies; panel $C$ is based on 159 replies. Car, cardiology; End, endocrinology; Gas, gastroenterology; Geri, geriatric medicine; Neu, neurology; Res, respiratory medicine; Rhe, rheumatology. 
study harder or concentrate more on neurology questions or cases to overcome any perceived extra difficulty. However, it is also possible that neurology could be handled worse than other specialties, and it would be worthwhile trying to test which of these two possibilities applies.

In the second part of this study, 78 SHOs discussed why they thought neurology was felt to be so difficult. They identified three major issues: the first centred on the need to know basic neurosciences, and neuroanatomy in particular; the second was the amount, type, and quality of the teaching given; and the third was the difficulty in carrying out a neurological examination. The main suggestion for tackling this problem was changes in teaching, with greater integration of clinical neurology and basic neurosciences, a plea for teachers to stress the most basic and simple concepts, and perhaps more and better teaching. This study is of potential importance for those involved in designing and running undergraduate neuroscience and neurology courses, and to a lesser extent those involved in the postgraduate training of doctors not destined to become neurologists.

There are strong traditions within neurological education, and we suspect there are widely differing opinions about the extent to which these can and should be altered. Would there be widespread support for a simplified "bare essentials" neuroscience course, aimed at giving only the clinically relevant parts of anatomy, physiology, pharmacology, and pathology, set in a continuous clinical context? There could be, and probably should be, an extended neuroscience course, with wider and deeper academic objectives.

Could the teaching of clinical neurology itself be simplified? The Association of British Neurologists' document, ${ }^{4}$ entitled "Teaching neurology in the 21 st century," stresses the importance of common disorders and common presenting symptoms, in contrast to the equivalent views set out in the USA. ${ }^{5}$ Some recent undergraduate textbooks are moving to simpler formats, avoiding both complex neuroscience and rare diagnoses. ${ }^{6-8}$

The critical question is whether the perception of neurology as such a difficult subject is a serious hindrance to students and non-career neurologists when they try to learn and practise the subject. If it is, and we suspect this to be the case, then the problem needs careful examination. It seems possible that neurologists have for too long tacitly enjoyed their subject's reputation as one for which only young Einsteins need apply. Though it is self evident that the brain is a more complex organ than, say, the skin or the joints or even the heart, we favour an analogy with driving-most people learn to drive safely with limited knowledge of how the engine works.

Neurological practice is also changing. The widespread availability of scanners means that acute diagnoses such as strokes, tumours, and intracerebral haemorrhages can now often be made by admitting general medical teams, with less reliance on the physical examination than in the past. Second, neurologists are becoming more involved in the treatment and rehabilitation of their patients, with a subtle shift in emphasis away from the details of clinical signs and diagnoses.

It is important to remember that in Britain most patients with neurological illnesses are still first seen by a general practitioner or a non-neurologically trained general medical team. We suspect that if patients are to receive optimal care from these nonneurologists then the teaching of neuroscience and neurology needs to be made as simple and accessible as possible right from the start at medical school, allowing trainees to build up their knowledge and clinical confidence as they proceed.

\section{CONCLUSIONS}

This survey confirms just how difficult neurology is perceived to be, not only by students but also by senior house officers and general practitioners. It highlights some of the reasons and suggests possible solutions. There needs to be a debate on whether major changes are needed in the way neuroscience and neurology are taught to medical students, to make the subject more accessible and more user friendly; otherwise we may frighten off another generation of non-neurologists.

\section{ACKNOWLEDGEMENTS}

We thank Ian Wilkinson and George Harwood for their helpful comments.

J Neurol Neurosurg Psychiatry 2002;72:557-559

\section{Authors' affiliations}

F Schon, Neurology Department, Atkinson Morley's Hospital, Copse Hill, London SW20 ONE, UK

P Hart, Neurology Department, Royal Free Hospital, Pond Street, London NW3 C Fernandez, Audit Department, Mayday Hospital, Thornton Heath, Surrey CRO 77 YE, UK

Correspondence to: Dr F Schon

\section{REFERENCES}

1 Editor's choice. Neurology for the masses. BM 1999:319:362.

2 Jozefowicz R. Neurophobia: the fear of neurology among medical students. Arch Neurol 1994;51:328-9.

3 Peterson S. Time for evidence based medical education. BM 1999;318:1223.

4 Association of British Neurologists. Teaching neurology in the 21 st century. London: Association of British Neurologists, 1994.

5 Charles PD, Scherokman B, Jozefowicz R. How much neurology should a medical student learn? A position statement of the AAN undergraduate education subcommittee. Acad Med 1999;74:23-6.

6 Donaghy M. Neurology. Oxford: Oxford University Press, 1997.

7 Wilkinson IMS. Essential neurology. Oxford: Blackwell Scientific Publications, 1998.

8 Fuller G. Neurological examination made easy. Edinburgh: Churchill Livingstone, 1998. 